\title{
Risk of Hospitalization for Adverse Drug Events in Women and Men: A Post Hoc Analysis of an Active Pharmacovigilance Study in Italian Emergency Departments
}

\author{
Giada Crescioli ${ }^{1,2}\left(\mathbb{D}\right.$, Ennio Boscia $^{1}$, Alessandra Bettiol ${ }^{3}$, Silvia Pagani ${ }^{4}$, Giulia Spada ${ }^{4}$, Giuditta Violetta Vighi ${ }^{4}$, \\ Roberto Bonaiuti ${ }^{1,5}$, Mauro Venegoni ${ }^{6}$, Giuseppe Danilo Vighi ${ }^{4}$, Alfredo Vannacci ${ }^{1,2,5,+}(\mathbb{D}$, \\ Niccolò Lombardi ${ }^{1,2, *},+(1)$ and on behalf of the MEREAFaPS Study Group $\ddagger$
}

Citation: Crescioli, G.; Boscia, E.; Bettiol, A.; Pagani, S.; Spada, G.; Vighi, G.V.; Bonaiuti, R.; Venegoni, M.; Vighi, G.D.; Vannacci, A.; et al. Risk of Hospitalization for Adverse Drug Events in Women and Men: A Post Hoc Analysis of an Active Pharmacovigilance Study in Italian Emergency Departments. Pharmaceuticals 2021, 14, 678. https:// doi.org/10.3390/ph14070678

Academic Editor: Olivia Manfrini

Received: 12 June 2021

Accepted: 13 July 2021

Published: 15 July 2021

Publisher's Note: MDPI stays neutral with regard to jurisdictional claims in published maps and institutional affiliations.

Copyright: (c) 2021 by the authors. Licensee MDPI, Basel, Switzerland. This article is an open access article distributed under the terms and conditions of the Creative Commons Attribution (CC BY) license (https:// creativecommons.org/licenses/by/ $4.0 /)$.
1 Department of Neurosciences, Psychology, Drug Research and Child Health, Section of Pharmacology and Toxicology, University of Florence, 50139 Florence, Italy; giada.crescioli@unifi.it (G.C.); ennio.boscia@stud.unifi.it (E.B.); roberto.bonaiuti@unifi.it (R.B.); alfredo.vannacci@unifi.it (A.V.)

2 Tuscan Regional Center of Pharmacovigilance, 50122 Florence, Italy

3 Department of Experimental and Clinical Medicine, University of Florence, 50134 Florence, Italy; alessandra.bettiol@unifi.it

4 Internal Medicine, Medical Department, Vimercate Hospital, ASST di Vimercate, 20871 Vimercate, Italy; silvia.pagani@asst-brianza.it (S.P.); giulia.spada@asst-brianza.it (G.S.); giudittavioletta.vighi@asst-brianza.it (G.V.V.); giuseppedanilo.vighi@asst-vimercate.it (G.D.V.)

5 Joint Laboratory of Technological Solutions for Clinical Pharmacology, Pharmacovigilance and Bioinformatics, University of Florence, 50139 Florence, Italy

6 Pharmacology Unit, Department of Diagnostics and Public Health, University of Verona, 37100 Verona, Italy; mauro.venegoni@gmail.com

* Correspondence: niccolo.lombardi@unifi.it; Tel.: +39-055-27-58-206

+ These authors contributed equally to this manuscript.

$\ddagger$ Membership of the MEREAFaPS Study Group is provided in the Acknowledgments.

\begin{abstract}
This post hoc analysis of an Italian active pharmacovigilance study describes pharmacological differences of ADEs leading to emergency department (ED) visits and hospitalization in women and men. During the study period (January 2007-December 2018), 61,855 reports of ADEs leading to ED visits were collected. Overall, 30.6\% of ADEs resulted in hospitalization $(30 \%$ in women and $31 \%$ in men). Multivariate logistic regression showed that, among women, drug classes significantly associated with an increased risk of hospitalization were heparins (ROR 1.41, CI 1.13-176), antidepressants (ROR 1.12, CI 1.03-1.23) and antidiabetics (ROR 1.13, CI 1.02-1.24). Among men, only vitamin K antagonists (ROR 1.28, CI 1.09-1.50), opioids (ROR 1.30, CI 1.06-1.60) and digitalis glycosides (ROR 1.32, CI 1.09-1.59) were associated with a higher risk of hospitalization. Overall, older age, multiple suspected drugs and the presence of comorbidities were significantly associated with a higher risk of hospitalization. A significantly reduced risk of hospitalization was observed in both women and men experiencing an adverse event following immunization (ROR 0.36, CI 0.27-0.48 and 0.83, 0.42-0.74, respectively) compared to drugs. Results obtained from this real-world analysis highlight important aspects of drug safety between sexes.
\end{abstract}

Keywords: pharmacovigilance; clinical pharmacology; male; female; emergency department

\section{Introduction}

Women and men are characterized by significant differences in terms of adverse drug event (ADE) occurrence derived from a heterogeneous set of factors in which both intrinsic and extrinsic elements concur [1,2]. Among these: (a) differences between men and women in body-water, muscle/fat mass ratio, organ blood flow and function; (b) physiological aspects such as menopause, pregnancy and menstruation; (c) prevalence of diseases and, consequently, in prescription patterns; (d) the possible impact of genetics and hor- 
monal variations in response to drugs [3-6]. All these factors describe the complexity of gender pharmacology.

As for randomized clinical trials, exclusion of women and limitation in drug safety evaluation are well-known issues [7,8]. Moreover, characterization of ADEs leading to emergency department (ED) admission in men and women is still lacking. Estimates of ADE risk between the two sexes from observational real-world data, especially from active pharmacovigilance studies, may represent the best strategy to fill this gap [9].

The MEREAFaPS Study was the first national active pharmacovigilance study performed in Italy. The study was based on electronic ED medical records with detailed information on patient populations, which allowed for consideration of risk predictors and modifying factors of ADEs and ADE-related hospitalization, such as polypharmacy and comorbidity, as well as sociodemographic characteristics [9]. This post hoc analysis of data retrieved from the MEREAFaPS Study database aimed to analyze pharmacological characteristics of ADEs leading to ED visits in women and men and to estimate differences in risk of hospitalization by different suspected drug classes.

\section{Results}

\subsection{Case Characteristics}

Between 1 January 2007 and 31 December 2018, 61,855 reports of ADEs leading to ED visit were collected: 35,010 (56.6\%) ADE reports for women and 26,845 (43.4\%) for men.

Table 1 shows the characteristics of cases. Overall, the majority of patients were Caucasian adults aged 20-79 years, with a median age of 62.4 years for women and 63.8 years for men. In both men and women, the majority of reports were related to drugs, and $\geq 5$ suspected drugs (polypharmacy) were reported in $23.2 \%$ of ADE reports for women and $25.4 \%$ of ADE reports for men.

Table 1. Case characteristics.

\begin{tabular}{|c|c|c|c|}
\hline & \multicolumn{3}{|c|}{ ED Visits for ADEs } \\
\hline & $\begin{array}{c}\text { Women } \\
N=35,010(\%)\end{array}$ & $\begin{array}{c}\text { Men } \\
N=26,845(\%)\end{array}$ & $p$-Value \\
\hline \multicolumn{4}{|l|}{ Patient Age, Years } \\
\hline$\leq 5$ & $1510(4.3)$ & $1701(6.3)$ & \multirow{6}{*}{$<0.001$} \\
\hline $6-19$ & $1607(4.6)$ & $1194(4.5)$ & \\
\hline $20-64$ & $15,238(43.5)$ & $10,801(40.2)$ & \\
\hline $65-79$ & $8456(24.2)$ & $7610(28.4)$ & \\
\hline$\geq 80$ & $7880(22.5)$ & $5295(19.7)$ & \\
\hline Not Available & $319(0.9)$ & $244(0.9)$ & \\
\hline Median (IQR), Years & $62.4(39.6-78.9)$ & $63.8(40.8-77.9)$ & 0.108 \\
\hline \multicolumn{4}{|l|}{ Patients' Ethnicity } \\
\hline Asian & $467(1.3)$ & $425(1.6)$ & \multirow{5}{*}{0.005} \\
\hline Black or African American & $291(0.8)$ & $259(1.0)$ & \\
\hline Caucasian & $30,729(87.8)$ & $23,503(87.6)$ & \\
\hline Other & $102(0.3)$ & $54(0.2)$ & \\
\hline Not available & $3421(9.8)$ & $2604(9.7)$ & \\
\hline \multicolumn{4}{|l|}{ Type of Drug } \\
\hline Drug & $34,425(98.3)$ & $26,259(97.8)$ & \multirow{2}{*}{$<0.001$} \\
\hline Vaccine & $585(1.7)$ & $586(2.2)$ & \\
\hline \multicolumn{4}{|c|}{ No. of Suspected Drugs Involved in the ADE } \\
\hline 1 & $14,948(42.7)$ & $11,054(41.2)$ & \multirow{4}{*}{$<0.001$} \\
\hline 2 & $5937(17.0)$ & $4575(17.0)$ & \\
\hline $3-4$ & $5988(17.1)$ & $4409(16.4)$ & \\
\hline$\geq 5$ & $8137(23.2)$ & $6807(25.4)$ & \\
\hline
\end{tabular}


Table 1. Cont.

\begin{tabular}{|c|c|c|c|}
\hline & \multicolumn{3}{|c|}{ ED Visits for ADEs } \\
\hline & $\begin{array}{c}\text { Women } \\
N=35,010(\%)\end{array}$ & $\begin{array}{c}\text { Men } \\
N=26,845(\%)\end{array}$ & $p$-Value \\
\hline Most Frequently Reported Suspected ATC Drug Classes & $N=44,119$ & $N=34,242$ & \\
\hline Antithrombotic Agents (B01) & $7322(16.6)$ & $8732(15.5)$ & $<0.001$ \\
\hline Antibacterials (J01) & $7203(16.3)$ & $5186(15.2)$ & $<0.001$ \\
\hline Anti-inflammatory and Antirheumatic Products (M01) & $3805(8.6)$ & $2812(8.2)$ & 0.039 \\
\hline Psycholeptics (N05) & $3717(8.4)$ & $2052(6.0)$ & $<0.001$ \\
\hline Analgesics (N02) & $3559(8.1)$ & $1915(5.6)$ & $<0.001$ \\
\hline Diabetes agents (A10) & $3051(6.9)$ & $2991(8.7)$ & $<0.001$ \\
\hline \multicolumn{4}{|l|}{ Presence of a Suspected Drug with Parenteral Administration } \\
\hline No & $30,478(87.1)$ & $23,187(86.4)$ & \multirow{2}{*}{0.013} \\
\hline Yes & $4532(12.9)$ & $3658(13.6)$ & \\
\hline \multicolumn{4}{|l|}{ Concomitant Drugs } \\
\hline No & $18,221(52.1)$ & $13,691(51.0)$ & \multirow{2}{*}{0.010} \\
\hline Yes & $16,789(48.0)$ & $13,154(49.0)$ & \\
\hline \multicolumn{4}{|l|}{ No. of Concomitant Drugs * } \\
\hline 0 & $18,221(52.1)$ & $13,691(51.0)$ & \multirow{5}{*}{$<0.001$} \\
\hline 1 & $4100(11.7)$ & $2977(11.1)$ & \\
\hline 2 & $2980(8.5)$ & $2220(8.3)$ & \\
\hline $3-4$ & $4414(12.6)$ & $3343(12.5)$ & \\
\hline$\geq 5$ & $5295(15.1)$ & $4614(17.2)$ & \\
\hline Most Frequently Reported Concomitant ATC Drug Classes & $N=61,184$ & $N=50,843$ & \\
\hline Renin-Angiotensin System Inhibitors (C09) & $5943(9.7)$ & $5175(10.2)$ & 0.009 \\
\hline Drugs for Acid Related Disorders (A02) & $5063(8.3)$ & $4090(8.0)$ & 0.162 \\
\hline Diuretics (C03) & $4899(8.0)$ & $3987(7.8)$ & 0.311 \\
\hline Antithrombotic Agents (B01) & $4851(7.9)$ & $4273(8.4)$ & 0.004 \\
\hline Beta Blocking Agents (C07) & $4521(7.4)$ & $4040(8.0)$ & $<0.001$ \\
\hline \multicolumn{4}{|l|}{ Presence of Comorbidities } \\
\hline No & $22,261(63.6)$ & $16,684(62.2)$ & \multirow{2}{*}{$<0.001$} \\
\hline Yes & $12,749(36.4)$ & $10,161(37.9)$ & \\
\hline \multicolumn{4}{|l|}{ No. of Comorbidities } \\
\hline 0 & $22,261(63.6)$ & $16,684(62.2)$ & \multirow{4}{*}{$<0.001$} \\
\hline 1 & $6111(17.5)$ & $4641(17.3)$ & \\
\hline 2 & $2819(8.1)$ & $2243(8.4)$ & \\
\hline$\geq 3$ & 3819 (10.9) & 3277 (12.2) & \\
\hline \multicolumn{4}{|l|}{ Most Frequently Reported Comorbidities } \\
\hline Hypertension & $3892(14.2)$ & $3261(14.3)$ & \multirow{8}{*}{$<0.001$} \\
\hline Diabetes & $1391(5.0)$ & $1306(5.7)$ & \\
\hline Atrial Fibrillation & $1321(4.8)$ & $1207(5.3)$ & \\
\hline Allergic Disease & $1060(3.8)$ & $577(2.4)$ & \\
\hline Heart Disease & $1023(3.7)$ & $1413(6.1)$ & \\
\hline Depression & $1020(3.7)$ & $372(1.6)$ & \\
\hline Dyslipidaemia & $828(3.0)$ & $776(3.4)$ & \\
\hline Renal Failure & $808(2.9)$ & $943(4.09)$ & \\
\hline \multicolumn{4}{|l|}{ Presence of CAM } \\
\hline No & 34,558 (98.7) & $26,569(99.0)$ & \multirow{2}{*}{0.003} \\
\hline Yes & $452(1.3)$ & $276(1.0)$ & \\
\hline
\end{tabular}


Table 1. Cont.

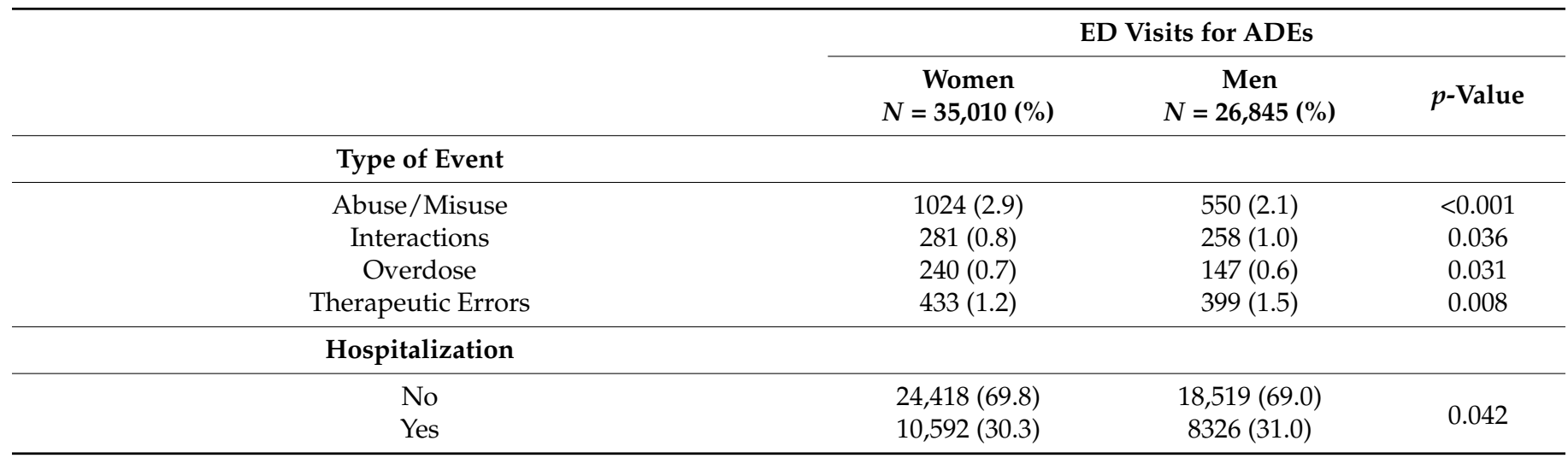

ADE: adverse drug event; ATC: anatomical therapeutic chemical; CAM: complementary and alternative medicines; ED: emergency department; IQR: interquartile range. * Number of concomitant drugs: "0" means that patient was not taking concomitant drugs, but only 1 or more suspected drugs; " 1 " means that patient was taking only 1 concomitant drug with at least 1 suspected drug; " 2 " or " $\geq 3$ " means that patient was taking 2 or more than 3 concomitant drugs with at least 1 suspected drug.

A statistically significant difference between the two sexes was observed for all the most frequently reported suspected ATC drug classes. For both sexes, the most frequently reported ATC classes of suspected drugs were antithrombotic agents $(16.6 \%$ in women and $15.5 \%$ in men), followed by antibacterials (16.3\% vs. $15.2 \%)$, anti-inflammatory and antirheumatic products ( $8.6 \%$ vs. $8.2 \%)$, psycholeptics $(8.4 \%$ vs. $6.0 \%)$, analgesics ( $8.1 \%$ vs. $5.6 \%)$ and antidiabetics (6.9\% vs. $8.7 \%)$.

The presence of concomitant drugs was reported in $48 \%$ of cases for women and $49 \%$ of cases for men. For both sexes, the most frequently reported ATC classes of concomitant drugs were renin-angiotensin system inhibitors ( $9.7 \%$ vs. $10.2 \%)$, followed by drugs for acid related disorders $(8.3 \%$ vs. $8.0 \%)$, diuretics $(8.0 \%$ vs. $7.8 \%)$, antithrombotic agents (7.9\% vs. $8.4 \%)$ and beta blocking agents $(7.4 \%$ vs. $8.0 \%)$.

Comorbidities were reported in $36.4 \%$ of cases for women and $37.9 \%$ of cases for men, with $10.9 \%$ of women and $12.2 \%$ of men affected by three or more concomitant diseases. For both sexes, the most reported comorbidity was hypertension, followed by diabetes and atrial fibrillation.

A statistically significant difference between the two sexes was observed for ADEs caused by abuse/misuse, drug-drug or herb-drug interactions, overdose and therapeutic errors.

Overall, 18,918 (30.5\%) ADEs caused patient hospitalization (30\% in women and 31\% in men, $p$-value $=0.042$ ).

\subsection{Hospitalization among Both Sexes}

A statistically significant increased risk of hospitalization was observed for both women and men exposed to antihemorrhagics, antianaemic and perfusion preparations (ROR 1.32, 95\% CI 1.14-1.52 and 1.33, 1.13-1.56, respectively); sedative or hypnotic agents (ROR 1.11, 1.01-1.21 and 1.15, 1.02-1.31), particularly benzodiazepines; antipsychotics (ROR 1.57, 1.38-1.79 and 1.44, 1.22-1.68); antiepileptics (ROR 1.23, 1.10-1.39 and 1.21, 1.05-1.38); diuretics (ROR 1.25, 1.15-1.36 and 1.16, 1.06-1.26) and, for diabetes agents, insulin (ROR 1.32, 1.12-1.55 and 1.19, 1.01-1.41) (Table 2).

A significantly reduced risk of hospitalization was observed for both women and men exposed to renin-angiotensin system inhibitors (ROR 0.89, 0.83-0.95 and 0.91, 0.84-0.98, respectively), particularly angiotensin II receptor blockers (plain and combinations) and ibuprofen (ROR 0.52, 0.36-0.76 and 0.56, 0.34-0.90) (Table 2). 
Table 2. Suspected drug classes and risk of hospitalization.

\begin{tabular}{|c|c|c|c|c|c|c|}
\hline & \multirow{2}{*}{\multicolumn{2}{|c|}{ ED Visits for ADEs }} & \multirow{2}{*}{\multicolumn{2}{|c|}{$\begin{array}{c}\text { ED Visits for ADEs } \\
\text { Resulting in Hospitalization }\end{array}$}} & \multirow{2}{*}{\multicolumn{2}{|c|}{$\begin{array}{c}\text { Adjusted ROR } \\
(95 \% \mathrm{CI})\end{array}$}} \\
\hline & & & & & & \\
\hline & $\begin{array}{c}\text { Women } \\
N=35,010(\%)\end{array}$ & $\begin{array}{c}\text { Men } \\
N=26,845(\%)\end{array}$ & $\begin{array}{c}\text { Women } \\
N=10,592(\text { row } \%)\end{array}$ & $\begin{array}{c}\text { Men } \\
N=8326(\text { row \%) }\end{array}$ & Women & Men \\
\hline \multicolumn{7}{|l|}{ Blood and Blood Forming Organs B } \\
\hline Anticoagulants (B01AA, B01AB, B01AE, B01AF, B01AX) & $1363(3.9)$ & $1044(3.9)$ & $672(57.0)$ & $507(43.0)$ & $1.13(1.00-1.26)$ & $1.14(1.00-1.30)$ \\
\hline Vitamin K Antagonists (B01AA) & $824(2.4)$ & $652(2.4)$ & $414(55.1)$ & $337(44.9)$ & $1.12(0.97-1.29)$ & $1.28(1.09-1.50)$ \\
\hline Factor Xa Inhibitors (B01AF) & $77(0.2)$ & $57(0.2)$ & $31(58.5)$ & $22(41.5)$ & $0.78(0.49-1.24)$ & $0.79(0.46-1.36)$ \\
\hline Unfractionated and Low-Molecular-Weight Heparins (B01AB) & $341(1.0)$ & $240(0.9)$ & $181(61.2)$ & $115(38.9)$ & $1.41(1.13-1.76)$ & $1.16(0.89-1.50)$ \\
\hline Antiplatelets (B01AC) & $3294(9.4)$ & $2927(10.9)$ & $1490(54.9)$ & $1226(45.1)$ & $0.92(0.85-1.00)$ & $0.81(0.74-0.88)$ \\
\hline Acetylsalicylic Acid (B01AC06) & $2679(7.7)$ & $2435(9.1)$ & $1206(54.8)$ & $995(45.2)$ & $0.94(0.86-1.02)$ & $0.79(0.72-0.87)$ \\
\hline $\begin{array}{l}\text { Platelet P2Y } Y_{12} \text { Receptor Antagonists (B01AC04, B01AC05, B01AC22, B01AC24, } \\
\text { B01AC25) }\end{array}$ & $689(2.0)$ & $705(2.6)$ & $317(51.5)$ & $299(48.5)$ & $0.91(0.78-1.07)$ & $0.81(0.69-0.95)$ \\
\hline Antihemorrhagics, Antianaemic and Perfusion Preparations (B02, B03, B05) & $828(2.4)$ & $680(2.5)$ & $438(54.3)$ & $369(45.7)$ & $1.32(1.14-1.52)$ & $1.33(1.13-1.56)$ \\
\hline \multicolumn{7}{|l|}{ Nervous System N } \\
\hline Analgesics (N02) & $1346(3.8)$ & $775(2.9)$ & $490(61.3)$ & $309(38.7)$ & $0.85(0.75-0.96)$ & $1.07(0.91-1.24)$ \\
\hline Opioid Analgesics (N02A) & $737(2.1)$ & 405 (1.5) & $323(60.7)$ & $209(39.3)$ & $0.94(0.81-1.10)$ & $1.30(1.06-1.60)$ \\
\hline Nonopioid Analgesics (N02B) & $615(1.8)$ & 387 (1.4) & $174(60.8)$ & $112(39.2)$ & $0.74(0.61-0.89)$ & $0.85(0.67-1.07)$ \\
\hline Benzodiazepines (N05BA, N05CD) & $2251(6.4)$ & $1008(3.8)$ & 1034 (68.6) & $473(31.4)$ & $1.12(1.02-1.22)$ & $1.15(1.01-1.32)$ \\
\hline Nonbenzodiazepine or Nonbarbiturate Sedatives (N05CF) & $200(0.6)$ & $99(0.4)$ & $92(63.5)$ & $53(36.6)$ & $1.04(0.78-1.38)$ & $1.33(0.89-1.99)$ \\
\hline Antidepressants (N06A) & $2385(6.8)$ & $1116(4.2)$ & $1101(68.0)$ & $519(32.0)$ & $1.12(1.03-1.23)$ & $1.08(0.95-1.23)$ \\
\hline Selective Serotonin Reuptake Inhibitors (N06AB) & $1489(4.3)$ & $669(2.5)$ & $705(69.7)$ & $306(30.3)$ & $1.19(1.07-1.33)$ & $1.07(0.91-1.26)$ \\
\hline Nonselective Serotonin Reuptake Inhibitors (N06AA) & $141(0.4)$ & $73(0.3)$ & 57 (62.6) & $34(37.4)$ & $1.01(0.71-1.42)$ & $1.27(0.79-2.04)$ \\
\hline Other Antidepressants (N06AF, N06AG, N06AX) & 917 (2.6) & $444(1.7)$ & $425(66.6)$ & $213(33.4)$ & $1.05(0.91-1.20)$ & $1.07(0.88-1.30)$ \\
\hline Antipsychotics (N05A) & $1051(3.0)$ & $673(2.5)$ & $569(61.7)$ & $353(38.3)$ & $1.57(1.38-1.79)$ & $1.44(1.22-1.68)$ \\
\hline Antiepileptics (N03) & $1353(3.9)$ & $960(3.6)$ & $637(58.2)$ & 457 (41.8) & $1.23(1.10-1.39)$ & $1.21(1.05-1.38)$ \\
\hline Anti-Parkinson Drugs (N04) & $395(1.1)$ & $340(1.3)$ & $192(54.7)$ & $159(45.3)$ & $1.06(0.86-1.30)$ & $0.99(0.80-1.24)$ \\
\hline Other Nervous System Agents (N01, N07) & $164(0.5)$ & $131(0.5)$ & $65(53.3)$ & $57(46.7)$ & $0.90(0.66-1.25)$ & $1.25(0.87-1.78)$ \\
\hline \multicolumn{7}{|l|}{ Anti-Infectives for Systemic Use J } \\
\hline Antibacterials (J01) & $740(2.1)$ & $591(2.2)$ & $227(52.8)$ & $203(47.2)$ & $0.79(0.67-0.94)$ & $0.92(0.77-1.10)$ \\
\hline Penicillins (J01C) & $260(0.7)$ & $221(0.8)$ & $61(45.5)$ & $73(54.5)$ & $0.62(0.46-0.83)$ & $1.05(0.78-1.41)$ \\
\hline Quinolones (J01M) & $190(0.5)$ & $179(0.7)$ & $76(55.1)$ & $62(44.9)$ & $1.08(0.79-1.46)$ & $0.76(0.56-1.05)$ \\
\hline Cephalosporins (J01D) & $121(0.4)$ & $89(0.3)$ & $45(52.3)$ & $41(47.7)$ & $1.00(0.68-1.48)$ & $1.30(0.84-2.01)$ \\
\hline Macrolides (J01F) & $122(0.4)$ & $81(0.3)$ & $41(63.1)$ & $24(36.9)$ & $0.90(0.61-1.34)$ & $0.83(0.50-1.36)$ \\
\hline Sulfamethoxazole and Trimethoprim (J01E) & $40(0.1)$ & $37(0.1)$ & $15(50.0)$ & $15(50.0)$ & $0.88(0.45-1.71)$ & $1.03(0.53-2.00)$ \\
\hline
\end{tabular}


Table 2. Cont.

\begin{tabular}{|c|c|c|c|c|c|c|}
\hline & \multirow{2}{*}{\multicolumn{2}{|c|}{ ED Visits for ADEs }} & \multirow{2}{*}{\multicolumn{2}{|c|}{$\begin{array}{c}\text { ED Visits for ADEs } \\
\text { Resulting in Hospitalization }\end{array}$}} & \multirow{2}{*}{\multicolumn{2}{|c|}{$\begin{array}{c}\text { Adjusted ROR } \\
(95 \% \mathrm{CI})\end{array}$}} \\
\hline & & & & & & \\
\hline & $\begin{array}{c}\text { Women } \\
N=35,010(\%)\end{array}$ & $\begin{array}{c}\text { Men } \\
N=26,845(\%)\end{array}$ & $\begin{array}{c}\text { Women } \\
N=10,592(\text { row } \%)\end{array}$ & $\begin{array}{c}\text { Men } \\
N=8326(\text { row \%) }\end{array}$ & Women & Men \\
\hline Other Antibacterials (J01A, J01B, J01G, J01R, J01X) & $60(0.2)$ & $29(0.1)$ & $14(51.9)$ & $13(48.2)$ & $0.54(0.29-0.99)$ & $1.40(0.66-2.96)$ \\
\hline Vaccines (J07) & $34(0.1)$ & $45(0.2)$ & $3(50.0)$ & $3(50.0)$ & $0.37(0.11-1.23)$ & $0.33(0.10-1.10)$ \\
\hline Antivirals and Antiretrovirals (J05) & $91(0.3)$ & $154(0.6)$ & $29(32.6)$ & $60(67.4)$ & $0.77(0.49-1.22)$ & $0.95(0.68-1.33)$ \\
\hline Other Anti-Infective Agents (J02, J04, J06) & $49(0.1)$ & $44(0.2)$ & $20(58.8)$ & $14(41.2)$ & $0.98(0.55-1.77)$ & $0.69(0.36-1.33)$ \\
\hline \multicolumn{7}{|l|}{ Cardiovascular System C } \\
\hline Renin-Angiotensin System Inhibitors (C09) & $5793(16.6)$ & 4998 (18.6) & $2531(53.7)$ & $2181(46.3)$ & $0.89(0.83-0.95)$ & $0.91(0.84-0.98)$ \\
\hline ACE Inhibitors (Plain and Combinations) (C09A, C09B) & $3255(9.3)$ & 3120 (11.6) & 1477 (51.4) & 1399(48.6) & $0.99(0.92-1.08)$ & $0.99(0.91-1.08)$ \\
\hline Angiotensin II Receptor Blockers (Plain and Combinations) (C09C, C09D) & $2620(7.5)$ & $1990(7.4)$ & $1092(56.4)$ & $843(43.6)$ & $0.84(0.77-0.91)$ & $0.88(0.80-0.98)$ \\
\hline Diuretics (C03) & 4004 (11.4) & 3203 (11.9) & $2100(56.3)$ & $1633(43.7)$ & $1.25(1.15-1.36)$ & $1.16(1.06-1.26)$ \\
\hline Low-Ceiling Diuretics (C03A, C03B) & $156(0.5)$ & $120(0.5)$ & $64(54.2)$ & $54(45.8)$ & $0.83(0.60-1.15)$ & $0.87(0.60-1.26)$ \\
\hline High-Ceiling Diuretics (C03C) & $3225(9.2)$ & $2745(10.2)$ & $1743(55.2)$ & $1413(44.8)$ & $1.29(1.18-1.41)$ & $1.16(1.05-1.27)$ \\
\hline Beta Blocking Agents (C07) & $4482(12.8)$ & 4004 (14.9) & $2000(52.6)$ & 1805 (47.4) & $0.92(0.85-0.99)$ & $0.94(0.87-1.03)$ \\
\hline Calcium Channel Blockers (C08) & $2213(6.3)$ & $1929(7.2)$ & 1033 (53.9) & $884(46.1)$ & $0.99(0.90-1.09)$ & $0.97(0.88-1.08)$ \\
\hline Antiarrhythmics (C01B) & $935(2.7)$ & $989(3.7)$ & $438(48.0)$ & $475(52.0)$ & $1.01(0.89-1.16)$ & $1.09(0.96-1.25)$ \\
\hline Lipid Modifying Agents (C10) & $3029(8.7)$ & 3361 (12.5) & $1311(46.3)$ & 1519 (53.7) & $0.84(0.77-0.91)$ & $0.92(0.85-1.01)$ \\
\hline Digitalis Glycosides (C01AA) & $813(2.3)$ & $463(1.7)$ & $425(62.8)$ & $252(37.2)$ & $1.10(0.95-1.27)$ & $1.32(1.09-1.59)$ \\
\hline \multicolumn{7}{|l|}{ Alimentary Tract and Metabolism A } \\
\hline Diabetes agents (A10) & $1991(5.7)$ & $1864(6.9)$ & $961(52.6)$ & $867(47.4)$ & $1.13(1.02-1.24)$ & $1.06(0.96-1.18)$ \\
\hline Insulins (A10A) & $662(1.9)$ & $641(2.4)$ & $345(52.3)$ & $315(47.7)$ & $1.32(1.12-1.55)$ & $1.19(1.01-1.41)$ \\
\hline Oral diabetes agents (A10B) & $1475(4.2)$ & $1360(5.1)$ & $692(53.2)$ & $610(46.9)$ & $1.04(0.93-1.16)$ & $0.96(0.86-1.08)$ \\
\hline Antiulcer and antacid agents (A02B, A02A) & $4899(14.0)$ & $3975(14.8)$ & $2319(55.3)$ & $1872(44.7)$ & $1.06(0.98-1.14)$ & $1.04(0.96-1.13)$ \\
\hline Antiemetics and antinauseants (A03F, A04) & $329(0.9)$ & $152(0.6)$ & $142(65.1)$ & $76(34.9)$ & $1.01(0.81-1.27)$ & $1.24(0.89-1.72)$ \\
\hline Antidiarrheals (A07) & $301(0.9)$ & $263(1.0)$ & $114(51.4)$ & $108(48.7)$ & $0.81(0.63-1.03)$ & $0.92(0.72-1.19)$ \\
\hline Drugs for constipation (A06) & $260(0.7)$ & $158(0.6)$ & $141(63.2)$ & $82(36.8)$ & $1.27(0.99-1.63)$ & $1.21(0.88-1.67)$ \\
\hline Stomatological preparations (A01) & $11(0.03)$ & $7(0.03)$ & $2(50.0)$ & $2(50.0)$ & $0.50(0.10-2.35)$ & $0.77(0.14-4.19)$ \\
\hline $\begin{array}{c}\text { Other gastrointestinal agents (A03, A05, A08, A09, A11, A12, A13, A14, A15, A16, } \\
\text { excluding A03F) }\end{array}$ & $928(2.7)$ & $576(2.2)$ & $472(61.9)$ & $291(38.1)$ & $1.19(1.04-1.37)$ & $1.18(0.99-1.40)$ \\
\hline
\end{tabular}


Table 2. Cont.

\begin{tabular}{|c|c|c|c|c|c|c|}
\hline & \multirow{2}{*}{\multicolumn{2}{|c|}{ ED Visits for ADEs }} & \multirow{2}{*}{\multicolumn{2}{|c|}{$\begin{array}{c}\text { ED Visits for ADEs } \\
\text { Resulting in Hospitalization }\end{array}$}} & \multirow{2}{*}{\multicolumn{2}{|c|}{$\begin{array}{c}\text { Adjusted ROR } \\
(95 \% \mathrm{CI})\end{array}$}} \\
\hline & & & & & & \\
\hline & $\begin{array}{c}\text { Women } \\
N=35,010(\%)\end{array}$ & $\begin{array}{c}\text { Men } \\
N=26,845(\%)\end{array}$ & $\begin{array}{c}\text { Women } \\
N=10,592(\text { row } \%)\end{array}$ & $\begin{array}{c}\text { Men } \\
N=8326(\text { row \%) }\end{array}$ & Women & Men \\
\hline \multicolumn{7}{|l|}{ Musculoskeletal system $\mathbf{M}$} \\
\hline Nonsteroidal Anti-Inflammatory Drugs (M01A) & $608(1.7)$ & $322(1.2)$ & $170(63.4)$ & $98(36.6)$ & $0.66(0.55-0.79)$ & $0.83(0.65-1.07)$ \\
\hline Ketoprofen (M01AE03, M01AE53) & $103(0.3)$ & $52(0.2)$ & $26(65.0)$ & $14(35.0)$ & $0.69(0.44-1.09)$ & $0.82(0.44-1.54)$ \\
\hline Ibuprofen (M01AE01, M01AE51) & $172(0.5)$ & $111(0.4)$ & $37(62.7)$ & $22(37.3)$ & $0.52(0.36-0.76)$ & $0.56(0.34-0.90)$ \\
\hline Diclofenac (M01AB05, M01AB55) & $88(0.3)$ & $50(0.2)$ & $36(65.5)$ & 19 (34.6) & $1.12(0.72-1.74)$ & $1.04(0.58-1.86)$ \\
\hline Ketorolac (M01AB15) & $55(0.2)$ & $35(0.1)$ & $13(54.2)$ & $11(45.8)$ & $0.47(0.25-0.89)$ & $0.72(0.34-1.48)$ \\
\hline Naproxen (M01AE02, M01AE52, M01AE56) & $21(0.06)$ & $8(0.03)$ & $6(75.0)$ & $2(25.0)$ & $0.65(0.25-1.73)$ & $0.61(0.12-3.12)$ \\
\hline Etoricoxib (M01AH05) & $57(0.2)$ & $16(0.06)$ & $23(76.7)$ & $7(23.3)$ & $0.83(0.48-1.42)$ & $1.11(0.41-3.04)$ \\
\hline Others (M01AA, M01AC, M01AG, M01AX) & $83(0.2)$ & $32(0.1)$ & $22(61.1)$ & $14(38.9)$ & $0.62(0.37-1.02)$ & $1.49(0.72-3.08)$ \\
\hline Muscle Relaxants (M03) & $112(0.3)$ & $79(0.3)$ & $33(55.9)$ & $26(44.1)$ & $0.69(0.46-1.05)$ & $0.72(0.44-1.17)$ \\
\hline Antigout Preparations (M04) & $1138(3.3)$ & $1388(5.2)$ & $616(47.1)$ & $692(52.9)$ & $1.20(1.06-1.37)$ & $1.08(0.96-1.22)$ \\
\hline Topical Products (M02) & $26(0.07)$ & $20(0.07)$ & $8(80.0)$ & $2(20.0)$ & $0.88(0.37-2.08)$ & $0.22(0.05-0.98)$ \\
\hline Bisphosphonates (M05) & $356(1.0)$ & $59(0.2)$ & $145(82.4)$ & $31(17.6)$ & $0.83(0.66-1.03)$ & $1.25(0.74-2.10)$ \\
\hline \multicolumn{7}{|l|}{ Antineoplastic and Immunomodulating Agents L } \\
\hline Immune Modulators (L03) & $22(0.06)$ & $23(0.09)$ & 10 (41.7) & $14(58.3)$ & $1.32(0.56-3.12)$ & $2.09(0.90-4.87)$ \\
\hline Endocrine Therapy (L02) & $220(0.6)$ & $143(0.5)$ & $93(57.1)$ & $70(42.9)$ & $1.05(0.80-1.39)$ & $0.98(0.70-1.37)$ \\
\hline \multicolumn{7}{|l|}{ Respiratory System R } \\
\hline Nasal, Throat, Cough and Cold Preparations (R01, R02, R05) & $192(0.6)$ & $179(0.7)$ & $50(49.5)$ & $51(50.5)$ & $0.65(0.47-0.91)$ & $0.70(0.50-0.98)$ \\
\hline Bronchodilators (R03) & $891(2.5)$ & $960(3.6)$ & $395(46.0)$ & $464(54.0)$ & $1.05(0.91-1.21)$ & $1.18(1.03-1.36)$ \\
\hline Antihistamines for Systemic Use (R06) & $258(0.7)$ & $149(0.6)$ & $83(63.4)$ & $48(36.6)$ & $0.75(0.57-0.98)$ & $0.82(0.57-1.17)$ \\
\hline \multicolumn{7}{|l|}{ Hormonal Preparations $\mathrm{H}$} \\
\hline Corticosteroids for Systemic Use (H02) & $1143(3.3)$ & $654(2.4)$ & $441(58.8)$ & $309(41.2)$ & $0.87(0.77-0.99)$ & $1.28(1.09-1.51)$ \\
\hline Thyroid Therapy (H03) & $2285(6.5)$ & $675(2.5)$ & $937(74.8)$ & $315(25.2)$ & $0.93(0.85-1.02)$ & $1.02(0.87-1.20)$ \\
\hline Other Hormonal Agents (H01, H04, H05) & $33(0.09)$ & $29(0.1)$ & $10(40.0)$ & $15(60.0)$ & $0.56(0.26-1.20)$ & $1.26(0.60-2.63)$ \\
\hline \multicolumn{7}{|l|}{ Genitourinary System and Sex Hormones G } \\
\hline Systemic and Vaginal Contraceptives (G01) & $14(0.04)$ & $6(0.02)$ & $6(75.0)$ & $2(25.0)$ & $1.29(0.43-3.89)$ & $0.91(0.16-5.23)$ \\
\hline Drugs Used in Benign Prostatic Hypertrophy (G04) & $60(0.2)$ & $1953(7.3)$ & $27(2.9)$ & $910(97.1)$ & $0.93(0.55-1.57)$ & $0.98(0.88-1.08)$ \\
\hline Other Gynaecological Agents and Sex Hormones (G02, G03) & $161(0.5)$ & $41(0.2)$ & $44(72.1)$ & $17(27.9)$ & $0.79(0.55-1.14)$ & $0.75(0.40-1.42)$ \\
\hline
\end{tabular}

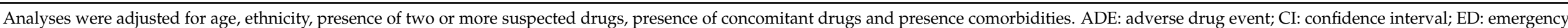
department; ROR: odds ratio. 


\subsection{Hospitalization Risks among Women}

A significantly increased risk of hospitalization was observed only for women when exposed to unfractionated and low-molecular-weight heparins (ROR 1.41, 1.13-1.76), antidepressants (ROR 1.12, 1.03-1.23) and diabetes agents (ROR 1.13, 1.02-1.24) (Table 2).

A statistically significant reduced risk of hospitalization was observed only for women when exposed to analgesics (ROR $0.85,0.75-0.96$ ), particularly nonopioid analgesics; antibacterials (ROR $0.79,0.67-0.94$ ), particularly penicillins; beta blocking agents (ROR 0.92 , 0.85-0.99); nonsteroidal anti-inflammatory drugs (ROR $0.66,0.55-0.79$ ), particularly ketorolac; and corticosteroids for systemic use (ROR 0.87, 0.77-0.99) (Table 2).

\subsection{Hospitalization Risks among Men}

A statistically significant increased risk of hospitalization was observed only for men when exposed to vitamin K antagonists (ROR 1.28, 1.09-1.50), opioid analgesics (ROR 1.30, 1.06-1.60), and digitalis glycosides (ROR 1.32, 1.09-1.59) (Table 2).

A significantly reduced risk of hospitalization was observed only for men when exposed to antiplatelets (ROR $0.81,0.74-0.88$ ), particularly acetylsalicylic acid and platelet $\mathrm{P} \mathrm{Y}_{12}$ receptor antagonists (Table 2).

\subsection{Predictors of Hospitalization}

A statistically significant increased risk of hospitalization was observed for both elderly women and men (ROR 1.35, 95\% CI 1.27-1.42 and 1.23, 1.16-1.31, respectively) compared to adults, in patients treated with more than one suspected drug (two suspected drugs ROR 1.98, 1.81-2.17 and 1.66, 1.50-1.84; three to four suspected drugs ROR 4.14, 3.66-4.69 and 2.92, 2.53-3.38; five or more drugs ROR 6.58, 5.66-7.66 and 4.15, 3.48-4.96), and in patients who presented concomitant conditions (ROR 1.51, 1.44-1.59 and 1.57, 1.48-1.66) (Table 3).

Table 3. Predictors of hospitalization among women and men expressed as reporting odds ratios.

\begin{tabular}{|c|c|c|c|c|}
\hline & \multicolumn{2}{|c|}{ Women } & \multicolumn{2}{|c|}{ Men } \\
\hline & $\begin{array}{c}\text { Crude ROR } \\
(95 \% \mathrm{CI})\end{array}$ & $\begin{array}{c}\text { Adjusted ROR } \\
(95 \% \text { CI })\end{array}$ & $\begin{array}{c}\text { Crude ROR } \\
(95 \% \mathrm{CI})\end{array}$ & $\begin{array}{c}\text { Adjusted ROR } \\
(95 \% \mathrm{CI})\end{array}$ \\
\hline \multicolumn{5}{|l|}{ Age Classes } \\
\hline Adults (20-64 Years) & 1 & 1 & 1 & 1 \\
\hline Children and Adolescents (0-19 Years) & $0.62(0.56-0.69)$ & $0.85(0.77-0.95)$ & $0.41(0.37-0.46)$ & $0.56(0.50-0.64)$ \\
\hline Elderly $(\geq 65$ Years $)$ & $2.00(1.90-2.10)$ & $1.35(1.27-1.42)$ & $1.79(1.69-1.88)$ & $1.23(1.16-1.31)$ \\
\hline \multicolumn{5}{|l|}{ Ethnicity } \\
\hline Caucasians & 1 & 1 & 1 & 1 \\
\hline Black or African American & $0.79(0.61-1.03)$ & $1.24(0.95-1.63)$ & $0.96(0.74-1.25)$ & $1.43(1.08-1.88)$ \\
\hline Asian & $0.57(0.46-0.72)$ & $0.89(0.70-1.13)$ & $0.61(0.48-0.76)$ & $0.93(0.73-1.19)$ \\
\hline Other & $0.78(0.50-1.22)$ & $1.09(0.69-1.73)$ & $0.63(0.33-1.19)$ & $0.86(0.44-1.67)$ \\
\hline \multicolumn{5}{|l|}{ AEFI } \\
\hline No & 1 & 1 & 1 & 1 \\
\hline Yes & $0.30(0.20-0.34)$ & $0.36(0.27-0.48)$ & $0.27(0.21-0.35)$ & $0.83(0.42-0.74)$ \\
\hline \multicolumn{5}{|l|}{ Number of Suspected Drugs } \\
\hline 1 & 1 & 1 & 1 & 1 \\
\hline 2 & $1.56(1.45-1.67)$ & $1.98(1.81-2.17)$ & $1.45(1.33-1.57)$ & $1.66(1.50-1.84)$ \\
\hline $3-4$ & $2.52(2.36-2.69)$ & $4.14(3.66-4.69)$ & $2.39(2.21-2.58)$ & $2.92(2.53-3.38)$ \\
\hline$\geq 5$ & $4.24(4.00-4.51)$ & $6.58(5.66-7.66)$ & $3.91(3.65-4.17)$ & $4.15(3.48-4.96)$ \\
\hline \multicolumn{5}{|l|}{ Concomitant Conditions } \\
\hline No & 1 & 1 & 1 & 1 \\
\hline Yes & $1.98(1.89-2.07)$ & $1.51(1.44-1.59)$ & $2.09(1.98-2.20)$ & $1.57(1.48-1.66)$ \\
\hline
\end{tabular}

Analyses were adjusted for age classes, ethnicity, type of drug, number of suspected drugs, number of concomitant drugs and presence of comorbidities. AEFI: adverse events following immunization; CI: confidence interval; ROR: reporting odds ratios. 
A significantly increased risk of hospitalization was observed only for men of Black or African American ethnicity (ROR 1.43, 1.08-1.88) compared to Caucasians (Table 3).

A significantly reduced risk of hospitalization was observed for both male and female children and adolescents (ROR $0.85,0.77-0.95$ and $0.56,0.50-0.64$, respectively) compared to adults, and for patients who experienced an adverse event following immunization (ROR $0.36,0.27-0.48$ and $0.83,0.42-0.74$ ) (Table 3).

\subsection{Adverse Events}

Table 4 shows the description of ADEs according to the most commonly reported suspected drug classes (see Table 1). A statistically significant difference between women and men was observed for the following ADEs: (1) haemorrhages, alteration of the international normalized ratio and unintentional or intentional overdose in patients exposed to anticoagulants and antiplatelets; (2) dermatologic reactions, gastrointestinal disturbances, neurological effects and anaphylaxis due to antibacterials; (3) dermatologic reactions, gastrointestinal disturbances, localized or peripheral edema and abuse or self-harm due to nonsteroidal anti-inflammatory drugs; (4) hypoglycaemia and gastrointestinal disturbances due to insulin and oral antidiabetic agents; (5) neurological effects, gastrointestinal disturbances, dermatologic reactions, localized or peripheral edema and unspecified hypersensitivity due to opioid and nonopioid analgesics. Details of each ADE manifestation are also reported (italics).

Table 4. Adverse events manifestation by most commonly reported suspected drug classes.

\begin{tabular}{|c|c|c|c|}
\hline & \multicolumn{3}{|c|}{ ED Visits for ADEs Resulting in Hospitalization } \\
\hline & $\begin{array}{c}\text { Women } \\
\text { No. of Preferred Terms } \\
N=47,937(\%)\end{array}$ & $\begin{array}{c}\text { Men } \\
\text { No. of Preferred Terms } \\
N=36,231(\%)\end{array}$ & $p$-Value \\
\hline \multicolumn{4}{|l|}{ Anticoagulants and Antiplatelets (B01A) } \\
\hline Haemorrhage & $4431(43.65)$ & $5719(51.45)$ & \multirow{8}{*}{$<0.001$} \\
\hline Epistaxis & $1476(14.52)$ & $2083(18.83)$ & \\
\hline Gastrointestinal & $858(8.46)$ & 1009 (9.13) & \\
\hline Genitourinary & $394(3.88)$ & $805(7.28)$ & \\
\hline Central Nervous System & $554(5.46)$ & $601(5.44)$ & \\
\hline Dermatologic & $54(0.53)$ & $59(0.54)$ & \\
\hline Pulmonary & $78(0.77)$ & $151(1.37)$ & \\
\hline Ophthalmic & $183(1.8)$ & $195(1.76)$ & \\
\hline Altered International Normalized Ratio & $822(8.10)$ & $661(5.97)$ & $<0.001$ \\
\hline Anaemia & $514(5.06)$ & $574(5.19)$ & 0.658 \\
\hline Unintentional or Intentional Overdose & $167(1.65)$ & $145(1.31)$ & 0.045 \\
\hline \multicolumn{4}{|l|}{ Antibacterials (J01) } \\
\hline Dermatologic Reactions & $6413(47.74)$ & $4441(47.73)$ & \multirow{5}{*}{$<0.001$} \\
\hline Urticaria & $1893(14.10)$ & $1315(14.14)$ & \\
\hline Localized or General Pruritus & $1661(12.38)$ & $1038(11.16)$ & \\
\hline Erythema & $1197(8.91)$ & $853(9.17)$ & \\
\hline Rash & $249(1.85)$ & $238(2.55)$ & \\
\hline Localized or Peripheral Edema & $1049(7.82)$ & $746(8.03)$ & \multirow[t]{2}{*}{0.571} \\
\hline Gastrointestinal Disturbances & $1532(11.43)$ & $913(9.83)$ & \\
\hline Nausea or Vomiting & $700(5.22)$ & $380(4.09)$ & \multirow{3}{*}{$<0.001$} \\
\hline Abdominal Pain & $397(2.96)$ & $247(2.66)$ & \\
\hline Diarrhoea & $351(2.62)$ & $237(2.55)$ & \\
\hline Unspecified Hypersensitivity & $636(4.74)$ & $414(4.46)$ & 0.311 \\
\hline Neurological Effects & $778(5.67)$ & $468(5.03)$ & 0.013 \\
\hline Respiratory Reactions & $728(5.43)$ & $486(5.22)$ & \multirow{3}{*}{0.514} \\
\hline Dyspnoea & $405(3.02)$ & $279(3.0)$ & \\
\hline Throat Tightness & $127(0.95)$ & $64(0.69)$ & \\
\hline Anaphylaxis & $117(0.87)$ & $118(1.27)$ & 0.004 \\
\hline
\end{tabular}


Table 4. Cont.

\begin{tabular}{|c|c|c|c|}
\hline & \multicolumn{3}{|c|}{ ED Visits for ADEs Resulting in Hospitalization } \\
\hline & $\begin{array}{c}\text { Women } \\
\text { No. of Preferred Terms } \\
N=47,937(\%)\end{array}$ & $\begin{array}{c}\text { Men } \\
\text { No. of Preferred Terms } \\
N=36,231(\%)\end{array}$ & $p$-Value \\
\hline \multicolumn{4}{|c|}{ Nonsteroidal Anti-Inflammatory Drugs (M01A) } \\
\hline Dermatologic Reactions & $2220(32.11)$ & $1571(30.01)$ & \multirow{6}{*}{0.012} \\
\hline Urticaria & $768(11.11)$ & $579(11.06)$ & \\
\hline Localized or General Pruritus & $572(8.28)$ & $385(7.35)$ & \\
\hline Erythema & $376(5.44)$ & $278(5.31)$ & \\
\hline Rash & $378(5.47)$ & $244(4.66)$ & \\
\hline Gastrointestinal Disturbances & $1256(18.18)$ & $862(16.51)$ & \\
\hline Abdominal Pain & $497(7.2)$ & $316(6.04)$ & \multirow{5}{*}{0.014} \\
\hline Nausea or Vomiting & $351(5.08)$ & $149(2.85)$ & \\
\hline Melena & $83(1.2)$ & $98(1.87)$ & \\
\hline Gastritis & $91(1.32)$ & $65(1.24)$ & \\
\hline Hematemesis & $58(0.84)$ & $65(1.24)$ & \\
\hline Localized or Peripheral Edema & $949(13.73)$ & $844(16.14)$ & $<0.001$ \\
\hline Unspecified Hypersensitivity & $235(3.4)$ & $198(3.79)$ & 0.262 \\
\hline Respiratory Reactions & $243(3.51)$ & $216(4.13)$ & 0.081 \\
\hline Abuse or Self-Harm & $100(1.45)$ & $52(0.99)$ & 0.026 \\
\hline \multicolumn{4}{|l|}{ Sedative or Hypnotic Agents (N05B, N05C) } \\
\hline Neurological Effects & $3721(57.44)$ & $2049(56.63)$ & \multirow{7}{*}{0.292} \\
\hline Drowsiness & $1226(18.93)$ & $607(16.7)$ & \\
\hline Altered Mental Status or Bradyphrenia & $504(7.78)$ & $270(7.43)$ & \\
\hline Loss of Consciousness & $179(2.76)$ & $103(3.04)$ & \\
\hline Bradykinesia & $196(3.03)$ & 119 (3.28) & \\
\hline Muscular Weakness & $116(2.56)$ & $101(2.78)$ & \\
\hline Presyncope or Syncope & $127(1.96)$ & $74(2.04)$ & \\
\hline \multicolumn{4}{|c|}{ Opioid and Nonopioid Analgesics (N02A, N02B) } \\
\hline Neurological Effects & $2124(28.29)$ & $866(23.21)$ & \multirow{8}{*}{$<0.001$} \\
\hline Muscular Weakness & $167(2.22)$ & $85(2.28)$ & \\
\hline Dizziness & $411(5.47)$ & $147(3.94)$ & \\
\hline Presyncope or Syncope & $258(3.44)$ & $97(2.6)$ & \\
\hline Drowsiness & $204(2.71)$ & $99(2.65)$ & \\
\hline Hyperhidrosis & $115(1.53)$ & $84(2.25)$ & \\
\hline Altered Mental Status & $246(3.28)$ & $117(3.14)$ & \\
\hline Headache & $107(1.43)$ & $32(0.86)$ & \\
\hline Gastrointestinal Disturbances & $2076(27.65)$ & $725(19.43)$ & \multirow{4}{*}{$<0.001$} \\
\hline Nausea or Vomiting & $1370(18.25)$ & $409(10.96)$ & \\
\hline Abdominal Pain & $508(6.77)$ & $199(5.33)$ & \\
\hline Constipation & $65(0.86)$ & $34(0.91)$ & \\
\hline Dermatologic Reactions & 1011 (13.47) & $686(18.39)$ & \multirow{5}{*}{$<0.001$} \\
\hline Urticaria & $343(4.57)$ & $249(6.68)$ & \\
\hline Localized or General Pruritus & $286(3.81)$ & $193(5.17)$ & \\
\hline Erythema & $214(2.85)$ & $125(3.35)$ & \\
\hline Rash & $168(2.24)$ & 119 (3.19) & \\
\hline Localized or Peripheral Edema & $380(5.07)$ & $248(6.64)$ & 0.001 \\
\hline Abuse or Self-Harm & $173(2.31)$ & $89(2.38)$ & 0.787 \\
\hline Respiratory Distress & $152(2.03)$ & $97(2.60)$ & 0.051 \\
\hline Unspecified Hypersensitivity & $106(1.41)$ & $91(2.44)$ & $<0.001$ \\
\hline
\end{tabular}


Table 4. Cont.

\begin{tabular}{|c|c|c|c|}
\hline & \multicolumn{3}{|c|}{ ED Visits for ADEs Resulting in Hospitalization } \\
\hline & $\begin{array}{c}\text { Women } \\
\text { No. of Preferred Terms } \\
N=47,937(\%)\end{array}$ & $\begin{array}{c}\text { Men } \\
\text { No. of Preferred Terms } \\
N=36,231(\%)\end{array}$ & $p$-Value \\
\hline \multicolumn{4}{|l|}{ Insulin and Oral Antidiabetic Agents (A10) } \\
\hline Hypoglycaemia (from Mild to Severe) & $1476(42.73)$ & $1530(46.8)$ & 0.001 \\
\hline Hypoglycaemia-Related Symptoms & $620(26.41)$ & $575(17.62)$ & \\
\hline Shock, Loss of Consciousness or Seizures & $260(7.52)$ & $276(8.45)$ & \\
\hline Altered Mental Status & $139(4.03)$ & $129(3.96)$ & 0.699 \\
\hline Presyncope or Syncope & $72(2.09)$ & $80(2.45)$ & \\
\hline Acidosis & $89(2.58)$ & $46(1.41)$ & \\
\hline Neurological Effects & $432(12.51)$ & $413(12.63)$ & \multirow{5}{*}{0.876} \\
\hline Drowsiness & $114(3.3)$ & $117(3.58)$ & \\
\hline Hyperhidrosis & $88(2.55)$ & $85(2.60)$ & \\
\hline Muscular Weakness & $61(1.77)$ & $41(1.25)$ & \\
\hline Aphasia, Dizziness or Tremor & $63(1.82)$ & $71(2.17)$ & \\
\hline Therapeutic Errors & $152(4.4)$ & $164(5.02)$ & 0.233 \\
\hline Gastrointestinal Disturbances & $151(4.37)$ & $98(3.01)$ & 0.003 \\
\hline
\end{tabular}

ADE: adverse drug event; ED: emergency department.

\subsection{Suspected Drugs}

Supplementary Table S1 shows the most commonly reported suspected drugs by patients' age. Overall, the most commonly reported drug was warfarin, followed by amoxicillin/clavulanate, acetylsalicylic acid, ketoprofen and ibuprofen. In patients aged $\leq 19$ years old, the most commonly reported drug was amoxicillin/clavulanate, followed by ibuprofen; amoxicillin, alone; hexavalent vaccine and paracetamol. Among elderly patients (age $\geq 65$ years), the most commonly reported drug was warfarin, followed by acetylsalicylic acid, amoxicillin/clavulanate, long-acting insulin glargine and furosemide.

\section{Discussion}

The primary aim of our study was to give an overview of differences in ADE-related hospitalization by the most frequently reported suspected drug classes in women and men in Italy. This post hoc analysis showed a higher frequency of ED admission in women, although for this group the frequency of hospitalization resulted lower than in men. This evidence are comparable to those already available in literature, both at the Italian $[10,11]$ and international level $[12,13]$.

Our data reported that men were exposed to polypharmacy ( $\geq 5$ suspected drugs along with $\geq 5$ concomitant drugs) more frequently than women at the time of ED admission, also presenting more than three comorbidities compared to women. This could be a possible explanation of the higher rate of hospitalization observed among men in our sample. In fact, at a global level, the proportion of serious and fatal reports also associated with hospitalization is higher for males [14].

It is noteworthy that vaccination safety was confirmed by the evidence that the majority of ADE reports were not associated to vaccines, nor was increased risk of hospitalization due to AEFI occurrence was observed among sexes. Although vaccines represent one of the most frequent cause of ADE in specific subgroups, such as children, their safety was confirmed by several observational studies and by health care authorities: adverse events following immunization are mostly nonserious and rarely cause ED visits and hospitalization [15].

According to the study by McHugh and colleagues [16], abuse/misuse and overdoses were more frequently reported among women. A possible explanation of these realworld evidences may be related to the higher prevalence of use of analgesics (i.e., opioids) and sedatives and hypnotics (i.e., benzodiazepines) among women in Italy [17], which 
could also be confirmed by the higher number of ADE reports related to these suspected drug classes for women in our sample. Furthermore, it is well-known that opioids and benzodiazepines have a high tolerance and dependence potential [18].

On the other hand, interactions (particularly drug-drug and drug-disease interactions) and therapeutic errors were more frequently reported among men. It is well-known that men, particularly the elderly or those exposed to polypharmacy as in our sample, take less care of themselves and, therefore, could be exposed to a greater risk of therapeutic errors, especially in absence of a caregiver, whose lacking is known to be more common among men [19]. Moreover, women more often act as caregivers, and the effect of having a caregiver appears less important for women compared to men [20].

Considering predictors of hospitalization, except for vaccines discussed above, our study confirms a higher risk of hospitalization for both sexes in old age ( $\geq 65$ years) and in subjects exposed to more than one suspected drug [21]. Moreover, even if genetic differences in drug response related to ethnicity are well established [22,23], social and economic status may have influenced the likelihood of seeing African American ethnicity associated with an increased risk of hospitalization for men. In fact, as reported by an Italian investigation, low education level, lack of employment and negative self-perceived economic resources were conditions associated with the risk of hospitalization, a longer hospital stay and greater recourse to urgent hospitalization [24].

As a final consideration, the majority of ADE manifestations observed in both sexes were represented by dose-dependent and preventable events (Type A reactions), which are usually associated with pharmacodynamic and pharmacokinetic properties of each suspected drug class, and by hypersensitivity events (Type B reactions), whose frequency is higher for antibacterials for systemic use [25].

\subsection{Drug Classes and Hospitalization Risk among Both Women and Men}

\subsubsection{Increased Hospitalization}

Scientific literature provides several studies concerning antipsychotic safety in women and men [26]. In much research, some side effects, such as weight gain, passivity, hypotension and hyperprolactinemia, are reported to be particularly problematic for women [27]. Nevertheless, in our sample, both women and men are at risk of hospitalization if exposed to antipsychotics. In fact, men and women may experience different ADEs: metabolic abnormalities, hypertension and cardiovascular risk are more frequently reported in men, while women are at a higher risk for gaining weight, developing diabetes and needing laxatives [28].

According to Landmark and Johannessen, there are no suggestion of sex-related influences on antiepileptics pharmacokinetics. In particular, sex does not influence antiepileptics efficacy and safety to a clinically relevant degree. Nevertheless, the use of enzyme inducers or inhibitors, such as carbamazepine or valproate, may be considered with caution, regardless of the patient's sex [29].

In contrast with our results, the German Pharmacovigilance Project found higher rates of hospitalization due to electrolyte disturbances and arrhythmias in women than in men treated with diuretics. According to the authors, even if dose adjustments are not required for patient's sex, diuretics elimination in women is reduced, leading to a higher frequency of hyponatraemia and hypokalaemia [30]. Results highlighted by our analysis may derive from a country-specific prescription patterns of diuretics, particularly high-ceiling ones. In fact, in the last report of the Italian Medicine Agency, no relevant differences were observed in diuretics prevalence of use in the general population [17]. Moreover, the clinical impact of concomitant diseases on hospitalization risk should also be considered.

To date, few studies focused on sex differences in the treatment of diabetes mellitus (Type 2) [31]. In line with our results, examination of the antidiabetic ADE pairs by drug class showed high rates of GLP-1RA-, insulin-, and SGLT2i-related reporting in both women and men. Explanations for possible different ADEs reporting by gender in diabetic subjects should be further explored [32,33]. 


\subsubsection{Decreased Hospitalization}

Results regarding the risk of hospitalization related to the use of renin-angiotensin system inhibitors and angiotensin II receptor blockers obtained in our sample are strengthened by the evidence of no sex-related differences in the pharmacokinetics of these drugs. Even if the premenopausal cardio-protective effects of estrogen may result in part from renin-angiotensin-aldosterone system inhibition, the efficacy and safety of medications acting on this system seems not to be imbalanced between the two sexes [30].

Compared with other nonsteroidal anti-inflammatory agents, ibuprofen shows a favourable safety profile. The majority of ADEs may be described as gastrointestinal and cardiovascular, but their incidence is relatively rare in both women and men [34]. Moreover, the menstrual cycle did not affect the pharmacokinetics of S-ibuprofen or R-ibuprofen, and only the concomitant administration of oral contraceptives may lead to a higher clearance. Nevertheless, the clinical impact of such interaction seems of little relevance [35].

\subsection{Drug Classes and Hospitalization Risk among Women \\ 3.2.1. Increased Hospitalization}

Our results regarding unfractionated and low-molecular-weight heparins are confirmed by Blanco-Molina et al., who showed an increased rate of major bleeding events in women, irrespective of the active principle used. The authors attributed the higher rate of bleeding in women to their older age, lower body weight or to the higher dosage, which may contribute to reach higher plasma concentration of heparins and increase the risk of ADEs [30,36].

Despite depression's prevalence in women, the vast majority of research focused on depression has been dedicated to studying males. Different gastric environment, slower gastric emptying and longer colonic transit observed in women can increase antidepressants absorption. In addition, a higher percentage of adipose tissue in women can prolong the half-life of lipophilic drugs. Differences in metabolism or clearance may contribute to higher plasma concentrations in women, and estrogen is a substrate for some of the same cytochrome P450 isozymes as well as antidepressants, possibly shifting their metabolism [30]. Thus, women show a decreased tolerability of such antidepressants, which could lead to dizziness, nausea, abnormal vision, constipation and somnolence [37].

While insulins increased hospitalization risk both in women and men, in our sample, oral antidiabetics related-ADEs were more frequently reported only in women. This is in line with previous evidences that some antidiabetics (i.e., thiazolidinediones) double the risk of fractures among diabetic women but not among men [30]. However, in the study by Rodenburg et al., hypoglycaemic coma due to insulin and antidiabetic agents were more frequent in men [38], whereas Hendriksen and colleagues found that hypoglycaemia was significantly lower in women than in men [13].

\subsubsection{Decreased Hospitalization}

Concerning nonopioid analgesics, mainly represented by paracetamol in our sample, we observed a reduced risk of hospitalization in women compared to men. This is in contrast with the review by Tamargo et al., which reported that paracetamol overdose and consequent acute liver failure are more common in women. In fact, men show lower plasma levels and higher clearance due to increased activity of the glucuronidation pathway [30].

Several studies from United States and Europe showed that antibiotics prescription is higher in women, although well-known risk factors for bacterial infections (i.e., alcohol drinking, smoking and obesity) are more prevalent in men [39,40]. Despite this, our analysis showed a lower risk of hospitalization due to antibiotic-related ADEs in this group. It can be assumed that women prescribed with antibiotics are more likely to receive appropriate prescriptions and to follow their therapy correctly [41].

Estrogen and progesterone inhibit the cardiac expression of $\beta 1$-adrenoceptors and reduce $\beta$-adrenergic-mediated stimulation. This cardio-protective effect may lead to sexspecific differences in beta blockers pharmacodynamics [40]. This evidence may explain 
our results concerning beta blockers safety profile in women. In fact, in women, more ADEs were observed for CYP 2D6-dependent beta blockers (i.e., metoprolol, carvedilol, nebivolol and propranolol), suggesting a possible increasing in hospitalization risk in case of drug-drug or drug-herb interactions [42].

Women receive diuretics more often than men, suggesting possible sex-related differences in the treatment of hypertension [40]. In contrast with our analysis, Rodenburg et al. found that women were more frequently admitted to the hospital with an ADE related to high-ceiling and low-ceiling diuretics and cardiotonic glycosides than men [43]. However, no information was reported in this study respect to hospitalization. Therefore, we cannot exclude that, although women are more exposed to diuretics and are admitted more frequently to ED, ADE seriousness in women may not require hospitalization.

\subsection{Drug Classes and Hospitalization Risk among Men}

\subsubsection{Increased Hospitalization}

As a result of their mechanism of action, anticoagulants cause different types of haemorrhages. Rodenburg et al. showed that the risk of a hospital admission differs between women and men for different types of bleeding. As in our study, men were more at risk for ED visits, in particular caused by unspecified and recurrent and persistent haematuria, haemoptysis and subdural haemorrhage [13]. Warfarin efficacy in reducing the risk of thromboembolism in women and men did not differ and did not pose women at a greater risk of major haemorrhagic complications. Women had more minor bleeding complications than men did, and they require less mg per week than men to maintain a therapeutic International Normalized Ratio, with older women requiring the lowest doses [40]. Another study confirms that the risk of hospitalizations for bleeding in any specific form due to anticoagulants or salicylates use was significantly higher in men. In particular, hospitalizations for haematuria and haemoptysis were much more frequent in men than in women. Risk for ADE-related hospitalization varied per type of reaction. Where men seemed to have a higher risk of hospitalization for haematuria, haemoptysis, cerebral bleeding and bone fractures [44], women were at higher risk for anaemia [38]. Of note, these studies considered anticoagulants and antiplatelets as a unique drug class, even if their pharmacological properties are not comparable.

Regarding opioids, women experience more ADEs (i.e., nausea and vomiting, respiratory depression) despite smaller dose requirements for pain control [38,40]. In our sample, a higher risk of hospitalization was not observed in women but was in men, according to the study by Hendriksen et al., which showed a small difference in the number of hospital admission in favour of men [13].

From our analysis, risk of hospitalization due to cardiotonic glycosides was significantly increased in men. According to the evidences published in literature, women have higher serum digoxin concentrations due to reduced distribution volume and lower clearance that increases only during pregnancy [40]. When directly compared, cardiotonic glycosides are responsible for a twofold higher risk for ED visits in women than men [43] and accounted for a risk for women to be hospitalized with an ADE that was twice as high as for men [43]. Our study did not underline an increased risk for hospitalization in women but, in line with the above cited articles, highlighted a worse safety profile for cardiotonic glycosides in men.

\subsubsection{Decreased Hospitalization}

As for antiplatelets, the majority of studies available in literature on this topic calculated pooled risk for anticoagulants and antiplatelets as combined drug classes. Following this approach, ED visits for bleeding due to anticoagulants or salicylates use were significantly higher in men [38]. However, when antiplatelets, alone, were considered, more frequent and severe bleedings were observed in women [40]. Again, our study did not underline an increased risk for hospitalization in women but confirmed a better safety profile for antiplatelets in men. 


\subsection{Limitations and Strengths}

Main limitations and strengths of the MEREAFaPS Study have already been described $[9,45]$. For this specific post hoc analysis, limitations are mainly represented by the lack of information on drug use and regarding drug-drug combinations and interactions. Points of strength include minimization of reporting bias by investigating only ADE-related ED visits and hospitalization and the availability of individual patient information, which enabled us to adjust for several demographic and clinical variables. Finally, to the best of our knowledge, this is the first analysis of its kind investigating drug safety in women and men in the setting of the ED without a direct comparison between the two groups. This methodological approach avoids obtaining estimates biased by all the well-known biological differences between sexes.

\section{Materials and Methods}

This is a post hoc analysis performed on pharmacovigilance reports of suspected $\mathrm{AE}$ collected between 1 January 2007 and 31 December 2018 in the EDs participating to the MEREAFaPS Study [9,45-47].

As described in previous publications [48,49], all ADEs leading to ED visits were collected from the ED clinical charts and hospitalization data were collected from the hospitals discharge database. Patients who developed an ADE while in the ED were excluded. Trained monitors recorded: (1) patients' demographic characteristics; (2) patients' clinical status on ED visits; (3) suspected and concomitant drugs; (4) ADEs description.

Suspected and concomitant drugs were classified according to the Anatomical Therapeutic Chemical (ATC) classification system. ADE description according to diagnosis and symptoms was coded using the Medical Dictionary for Regulatory Activities (MedDRA) and organized by System Organ Class (SOC) and Preferred Term (PT) [48]. As already described in previous publications, "suspected" drugs were defined as those mainly associated with the reported ADE, while "concomitant" drugs as those described in the report form at the time of ADE manifestation, which were used by the patient concomitantly with the suspected drugs, but which were not considered directly associated with the ADE. Number of concomitant drugs " 0 " means that patient was not taking concomitant drugs, but only 1 or more suspected drugs; " 1 " means that patient was taking only 1 concomitant drug with at least 1 suspected drug; " 2 " or " $\geq 3$ " means that patient was taking 2 or more than 3 concomitant drugs with at least 1 suspected drug.

Descriptive statistics were used to summarize data. Categorical data were reported as frequencies and percentages and compared using the chi-square test, whereas continuous data were reported as median values with the related interquartile ranges (IQR) and compared using the Mann-Whitney test. Univariate logistic regression was used to estimate the reporting odds ratios (RORs) of hospitalization with 95\% confidence intervals (CIs). In order to reduce bias due to biological differences between women and men, comparisons were made within each sex group rather than between the two sexes. For example, estimating the hospitalization risk associated to anticoagulants, women exposed to these suspected drugs were compared to not exposed ones. The same contingency was built for men. Multivariate logistic regression was performed and adjusted for: age, ethnicity, number of suspected drugs, presence of concomitant drugs and presence of concomitant conditions. All results were considered to be statistically significant at $p$-value $<0.05$. Data management and statistical analysis were carried out using STATA 16.1.

The coordinating centre of Tuscany Region (Italy) approved MEREAFaPS Study (Notification number 1225-21 December 2009), and the local institutional ethics committee approved the MEREAFaPS Study (Study number 3055/2010, Protocol number 452886 August 2014) according to the legal requirements concerning observational studies. Due to the retrospective nature of the present study and data anonymization, patient consent to participate was not required. 


\section{Conclusions}

Results obtained from this real-world analysis highlight important aspects of drug safety between sexes. Healthcare professionals, particularly physicians operating in ED and clinical pharmacologists, should always consider differences in drug safety among women and men, considering a personalized approach for each group in terms of prescription appropriateness and ADE management and prevention.

Supplementary Materials: The following are available online at https:/ / www.mdpi.com/article/10 .3390/ph14070678/s1, Table S1: Most commonly reported suspected drugs by patient's age.

Author Contributions: Conceptualization, N.L. and G.C.; methodology, A.B.; formal analysis, A.B. and R.B.; data curation, G.S., G.V.V. and E.B.; writing-original draft preparation, G.C. and S.P.; writing-review and editing, G.C., S.P. and N.L.; supervision, A.V., G.D.V. and M.V. All authors have read and agreed to the published version of the manuscript.

Funding: This study was funded by a research grant from the AIFA (the Italian Medicines Agency), Rome, Italy, Tuscan County resolution DGRT 790/2016 All. C. The funder of the study had no role in the collection, analysis or interpretation of data, nor in the writing of the report, nor in the decision to submit the article for publication.

Institutional Review Board Statement: The coordinating centre of Tuscany Region (Italy) approved MEREAFaPS Study (Notification number 1225-21 December 2009), and the local institutional ethics committee approved the MEREAFaPS Study (Study number 3055/2010, Protocol number 45288-6 August 2014) according to the legal requirements concerning observational studies.

Informed Consent Statement: Due to the retrospective nature of the present study and data anonymization, patient consent to participate was not required.

Data Availability Statement: Data that support the findings of this study are available upon reasonable request from the corresponding author, N.L.

Acknowledgments: Members of the MEREAFaPS Study group who provided patient data for this study: Maria Luisa Aiezza (Naples), Alessandra Bettiol (Florence), Daria Bettoni (Brescia), Corrado Blandizzi (Pisa), Roberto Bonaiuti (Florence), Valentina Borsi (Florence), Annalisa Capuano (Naples), Errica Cecchi (Prato), Irma Convertino (Pisa), Giada Crescioli (Florence), Martina Del Lungo (Florence), Cristina Di Mauro (Naples), Gabriella Farina (Milan), Sara Ferraro (Pisa), Annamaria Fucile (Naples), Elena Galfrascoli (Milan), Elisabetta Geninatti (Turin), Linda Giovannetti (Florence), Luca Leonardi (Pisa), Rosa Liccardo (Naples), Niccolò Lombardi (Florence), Anna Marra (Ferrara), Eleonora Marrazzo (Turin), Giovanna Monina (Gallarate), Alessandro Mugelli (Florence), Silvia Pagani (Vimercate), Maria Parrilli (Florence), Concetta Rafaniello (Naples), Francesco Rossi (Naples), Marco Rossi (Siena), Stefania Rostan (Naples), Marco Ruocco (Vimercate), Marita Sironi (Vimercate), Giulia Spada (Vimercate), Liberata Sportiello (Naples), Marco Tuccori (Pisa), Alfredo Vannacci (Florence), Mauro Venegoni (Vimercate), Giuditta Violetta Vighi (Vimercate), Giuseppe Danilo Vighi (Vimercate).

Conflicts of Interest: The authors declare no conflict of interest.

\section{References}

1. Franconi, F.; Campesi, I. Pharmacogenomics, pharmacokinetics and pharmacodynamics: Interaction with biological differences between men and women. Br. J. Pharmacol. 2014, 171, 580-594. [CrossRef]

2. Farkouh, A.; Baumgartel, C.; Gottardi, R.; Hemetsberger, M.; Czejka, M.; Kautzky-Willer, A. Sex-related differences in drugs with anti-inflammatory properties. J. Clin. Med. 2021, 10, 441. [CrossRef] [PubMed]

3. Tran, C.; Knowles, S.R.; Liu, B.A.; Shear, N.H. Gender differences in adverse drug reactions. J. Clin. Pharmacol. 1998, 38, 1003-1009. [CrossRef] [PubMed]

4. Zopf, Y.; Rabe, C.; Neubert, A.; Janson, C.; Brune, K.; Hahn, E.G.; Dormann, H. Gender-based differences in drug prescription: Relation to adverse drug reactions. Pharmacology 2009, 84, 333-339. [CrossRef] [PubMed]

5. Schwartz, J.B. The influence of sex on pharmacokinetics. Clin. Pharmacokinet. 2003, 42, 107-121. [CrossRef]

6. Kashuba, A.D.; Nafziger, A.N. Physiological changes during the menstrual cycle and their effects on the pharmacokinetics and pharmacodynamics of drugs. Clin. Pharmacokinet. 1998, 34, 203-218. [CrossRef] [PubMed]

7. Shields, K.E.; Lyerly, A.D. Exclusion of pregnant women from industry-sponsored clinical trials. Obstet. Gynecol. 2013, 122, 1077-1081. [CrossRef] 
8. Singh, S.; Loke, Y.K. Drug safety assessment in clinical trials: Methodological challenges and opportunities. Trials 2012, 13, 138. [CrossRef]

9. Lombardi, N.; Crescioli, G.; Bettiol, A.; Tuccori, M.; Capuano, A.; Bonaiuti, R.; Mugelli, A.; Venegoni, M.; Vighi, G.D.; Vannacci, A.; et al. Italian emergency department visits and hospitalizations for outpatients' adverse drug events: 12-year active pharmacovigilance surveillance (The MEREAFaPS Study). Front. Pharmacol. 2020, 11, 412. [CrossRef]

10. Giovannella Baggio, W.M. The Italian law on gender medicine: A reality and a hope. Ital. J. Gend. Specif. Med. 2019, 5, 105-107. [CrossRef]

11. Giovannella Baggio, W.M. The Italian Journal of Gender-Specific Medicine and the Italian Gender Medicine Network. Ital. J. Gend. Specif. Med. 2018, 4, 1-2. [CrossRef]

12. de Vries, S.T.; Denig, P.; Ekhart, C.; Burgers, J.S.; Kleefstra, N.; Mol, P.G.M.; van Puijenbroek, E.P. Sex differences in adverse drug reactions reported to the National Pharmacovigilance Centre in the Netherlands: An explorative observational study. Br. J. Clin. Pharmacol. 2019, 85, 1507-1515. [CrossRef] [PubMed]

13. Hendriksen, L.C.; van der Linden, P.D.; Lagro-Janssen, A.L.M.; van den Bemt, P.; Siiskonen, S.J.; Teichert, M.; Kuiper, J.G.; Herings, R.M.C.; Stricker, B.H.; Visser, L.E. Sex differences associated with adverse drug reactions resulting in hospital admissions. Biol. Sex Differ. 2021, 12, 34. [CrossRef] [PubMed]

14. Watson, S.; Caster, O.; Rochon, P.A.; den Ruijter, H. Reported adverse drug reactions in women and men: Aggregated evidence from globally collected individual case reports during half a century. EClinicalMedicine 2019, 17, 100188. [CrossRef] [PubMed]

15. Scavone, C.; Rafaniello, C.; Brusco, S.; Bertini, M.; Menditto, E.; Orlando, V.; Trama, U.; Sportiello, L.; Rossi, F.; Capuano, A. Did the New Italian Law on Mandatory Vaccines Affect Adverse Event Following Immunization's Reporting? A Pharmacovigilance Study in Southern Italy. Front. Pharmacol. 2018, 9, 1003. [CrossRef]

16. McHugh, R.K.; Geyer, R.B.; Chase, A.R.; Griffin, M.L.; Bogunovic, O.; Weiss, R.D. Sex differences in benzodiazepine misuse among adults with substance use disorders. Addict. Behav. 2021, 112, 106608. [CrossRef]

17. Farmaco, A.A.I.d. L'uso dei farmaci in Italia-Rapporto Nazionale Anno 2019. Available online: https://www.aifa.gov.it/ documents /20142/1205984/rapporto-osmed-2019.pdf (accessed on 12 June 2021).

18. Azevedo, K.; Johnson, M.; Wassermann, M.; Evans-Wall, J. Drugs of Abuse-Opioids, Sedatives, Hypnotics. Crit. Care Clin. 2021, 37, 501-516. [CrossRef]

19. Talamantes, M.A.; Cornell, J.; Espino, D.V.; Lichtenstein, M.J.; Hazuda, H.P. SES and ethnic differences in perceived caregiver availability among young-old Mexican Americans and non-Hispanic whites. Gerontologist 1996, 36, 88-99. [CrossRef]

20. Blackburn, J.; Albright, K.C.; Haley, W.E.; Howard, V.J.; Roth, D.L.; Safford, M.M.; Kilgore, M.L. Men Lacking a Caregiver Have Greater Risk of Long-Term Nursing Home Placement After Stroke. J. Am. Geriatr. Soc. 2018, 66, 133-139. [CrossRef]

21. Chang, T.I.; Park, H.; Kim, D.W.; Jeon, E.K.; Rhee, C.M.; Kalantar-Zadeh, K.; Kang, E.W.; Kang, S.W.; Han, S.H. Polypharmacy, hospitalization, and mortality risk: A nationwide cohort study. Sci. Rep. 2020, 10, 18964. [CrossRef]

22. Baehr, A.; Pena, J.C.; Hu, D.J. Racial and Ethnic Disparities in Adverse Drug Events: A Systematic Review of the Literature. J. Racial Ethn. Health Disparit. 2015, 2, 527-536. [CrossRef]

23. Ortega, V.E.; Meyers, D.A. Pharmacogenetics: Implications of race and ethnicity on defining genetic profiles for personalized medicine. J. Allergy Clin. Immunol. 2014, 133, 16-26. [CrossRef]

24. Petrelli, A.; Di Napoli, A.; Demuru, E.; Ventura, M.; Gnavi, R.; Di Minco, L.; Tamburini, C.; Mirisola, C.; Sebastiani, G. Socioeconomic and citizenship inequalities in hospitalisation of the adult population in Italy. PLoS ONE 2020, 15, e0231564. [CrossRef] [PubMed]

25. Edwards, I.R.; Aronson, J.K. Adverse drug reactions: Definitions, diagnosis, and management. Lancet 2000, 356, 1255-1259. [CrossRef]

26. Trifiro, G.; Spina, E.; Brignoli, O.; Sessa, E.; Caputi, A.P.; Mazzaglia, G. Antipsychotic prescribing pattern among Italian general practitioners: A population-based study during the years 1999-2002. Eur. J. Clin. Pharmacol. 2005, 61, 47-53. [CrossRef]

27. Seeman, M.V. Gender differences in the prescribing of antipsychotic drugs. Am. J. Psychiatry 2004, 161, 1324-1333. [CrossRef]

28. Alberich, S.; Fernandez-Sevillano, J.; Gonzalez-Ortega, I.; Usall, J.; Saenz, M.; Gonzalez-Fraile, E.; Gonzalez-Pinto, A. A systematic review of sex-based differences in effectiveness and adverse effects of clozapine. Psychiatry Res. 2019, 280, 112506. [CrossRef]

29. Johannessen Landmark, C.; I Johannessen, S. Pharmacotherapy in epilepsy—Does gender affect safety? Expert Opin. Drug Saf. 2016, 15, 1-4. [CrossRef] [PubMed]

30. Tamargo, J.; Rosano, G.; Walther, T.; Duarte, J.; Niessner, A.; Kaski, J.C.; Ceconi, C.; Drexel, H.; Kjeldsen, K.; Savarese, G.; et al Gender differences in the effects of cardiovascular drugs. Eur. Heart J. Cardiovasc. Pharmacother. 2017, 3, 163-182. [CrossRef] [PubMed]

31. Arnetz, L.; Ekberg, N.R.; Alvarsson, M. Sex differences in type 2 diabetes: Focus on disease course and outcomes. Diabetesmetabolic Syndr. Obes. Targets Ther. 2014, 7, 409-420. [CrossRef]

32. Joung, K.I.; Jung, G.W.; Park, H.H.; Lee, H.; Park, S.H.; Shin, J.Y. Gender differences in adverse event reports associated with antidiabetic drugs. Sci. Rep. 2020, 10, 17545. [CrossRef]

33. Jovanovic, L. Sex differences in insulin dose and postprandial glucose as BMI increases in patients with type 2 diabetes. Diabetes Care 2009, 32, e148. [CrossRef]

34. Varrassi, G.; Pergolizzi, J.V.; Dowling, P.; Paladini, A. Ibuprofen Safety at the Golden Anniversary: Are all NSAIDs the Same? A Narrative Review. Adv. Ther. 2020, 37, 61-82. [CrossRef] 
35. Madla, C.M.; Gavins, F.K.H.; Merchant, H.; Orlu, M.; Murdan, S.; Basit, A.W. Let's Talk About Sex: Differences in Drug Therapy in Males and Females. Adv. Drug Deliv. Rev. 2021. [CrossRef]

36. Blanco-Molina, A.; Enea, I.; Gadelha, T.; Tufano, A.; Bura-Riviere, A.; Di Micco, P.; Bounameaux, H.; Gonzalez, J.; Villalta, J.; Monreal, M.; et al. Sex differences in patients receiving anticoagulant therapy for venous thromboembolism. Medicine 2014, 93, 309-317. [CrossRef]

37. LeGates, T.A.; Kvarta, M.D.; Thompson, S.M. Sex differences in antidepressant efficacy. Neuropsychopharmacology 2019, $44,140-154$. [CrossRef]

38. Rodenburg, E.M.; Stricker, B.H.; Visser, L.E. Sex-related differences in hospital admissions attributed to adverse drug reactions in the Netherlands. Br. J. Clin. Pharmacol. 2011, 71, 95-104. [CrossRef] [PubMed]

39. Schroder, W.; Sommer, H.; Gladstone, B.P.; Foschi, F.; Hellman, J.; Evengard, B.; Tacconelli, E. Gender differences in antibiotic prescribing in the community: A systematic review and meta-analysis. J. Antimicrob. Chemother. 2016, 71, 1800-1806. [CrossRef] [PubMed]

40. Orlando, V.; Monetti, V.M.; Moreno Juste, A.; Russo, V.; Mucherino, S.; Trama, U.; Guida, A.; Menditto, E. Drug Utilization Pattern of Antibiotics: The Role of Age, Sex and Municipalities in Determining Variation. Risk Manag. Healthc. Policy 2020, $13,63-71$. [CrossRef]

41. Smith, D.R.M.; Dolk, F.C.K.; Smieszek, T.; Robotham, J.V.; Pouwels, K.B. Understanding the gender gap in antibiotic prescribing: A cross-sectional analysis of English primary care. BMJ Open 2018, 8, e020203. [CrossRef]

42. Kalibala, J.; Pechere-Bertschi, A.; Desmeules, J. Gender Differences in Cardiovascular Pharmacotherapy-the Example of Hypertension: A Mini Review. Front. Pharmacol. 2020, 11, 564. [CrossRef] [PubMed]

43. Rodenburg, E.M.; Stricker, B.H.; Visser, L.E. Sex differences in cardiovascular drug-induced adverse reactions causing hospital admissions. Br. J. Clin. Pharmacol. 2012, 74, 1045-1052. [CrossRef]

44. Lucenteforte, E.; Bettiol, A.; Lombardi, N.; Mugelli, A.; Vannacci, A. Risk of bone fractures among users of oral anticoagulants: An administrative database cohort study. Eur. J. Intern. Med. 2017, 44, e30-e31. [CrossRef]

45. Crescioli, G.; Bettiol, A.; Bonaiuti, R.; Tuccori, M.; Rossi, M.; Capuano, A.; Pagani, S.; Spada, G.; Venegoni, M.; Vighi, G.D.; et al. Risk of Hospitalization Associated with Cardiovascular Medications in the Elderly Italian Population: A Nationwide Multicenter Study in Emergency Departments. Front. Pharmacol. 2020, 11, 611102. [CrossRef] [PubMed]

46. Pagani, S.; Lombardi, N.; Crescioli, G.; Vighi, G.V.; Spada, G.; Romoli, I.; Andreetta, P.; Capuano, A.; Marrazzo, E.; Marra, A.; et al. Analysis of fatal adverse drug events recorded in several Italian emergency departments (the MEREAFaPS study). Intern. Emerg. Med. 2021, 16, 741-748. [CrossRef] [PubMed]

47. Lombardi, N.; Bettiol, A.; Crescioli, G.; Ravaldi, C.; Bonaiuti, R.; Venegoni, M.; Vighi, G.D.; Mugelli, A.; Mannaioni, G.; Vannacci, A.; et al. Risk of hospitalisation associated with benzodiazepines and z-drugs in Italy: A nationwide multicentre study in emergency departments. Intern. Emerg. Med. 2020, 15, 1291-1302. [CrossRef]

48. Lombardi, N.; Crescioli, G.; Bettiol, A.; Marconi, E.; Vitiello, A.; Bonaiuti, R.; Calvani, A.M.; Masi, S.; Lucenteforte, E.; Mugelli, A.; et al. Characterization of serious adverse drug reactions as cause of emergency department visit in children: A 5-years active pharmacovigilance study. BMC Pharmacol. Toxicol. 2018, 19, 16. [CrossRef]

49. Lombardi, N.; Crescioli, G.; Bettiol, A.; Tuccori, M.; Rossi, M.; Bonaiuti, R.; Ravaldi, C.; Levi, M.; Mugelli, A.; Ricci, S.; et al. Vaccines Safety in Children and in General Population: A Pharmacovigilance Study on Adverse Events Following Anti-Infective Vaccination in Italy. Front. Pharmacol. 2019, 10, 948. [CrossRef] 